\title{
ON LINEAL ENTIRE FUNCTIONS OF $n$ COMPLEX VARIABLES ${ }^{1}$
}

\author{
T. S. MOTZKIN AND I. J. SCHOENBERG
}

1. Statement of problems and main results. A polynomial $P\left(z_{1}, \cdots, z_{n}\right)$ with real or complex coefficients is called lineal if it splits into a product of linear functions of the variables in the complex field of coefficients. Every polynomial $P\left(z_{1}\right)$, of one variable only, is lineal, by the fundamental theorem of algebra; however, for two or more variables lineality is a severe restriction. As an example we mention the cyclic determinant of order $n$ which is a lineal polynomial in the elements of its first row.

We say that a sequence of entire functions $f_{k}\left(z_{1}, \cdots, z_{n}\right)$ converges regularly to a function $f\left(z_{1}, \cdots, z_{n}\right)$, and write reg. $\lim f_{k}=f$, meaning thereby that the convergence holds uniformly in every bounded portion of space. Every entire function is a regular limit of polynomials, for instance the sections of its Taylor expansion. But what if we require the approximating polynomials to be lineal? More explicitly: What entire functions $f\left(z_{1}, \cdots, z_{n}\right)$ are regular limits of lineal polynomials? We call such functions lineal and denote their class by the symbol $\mathcal{L}^{(n)}$.

By a plane we mean the manifold defined by one linear equation in the variables. It seems plausible that a lineal function will vanish on planes only, by which we mean the following: If $f$ vanishes in a point, then it will vanish on a plane passing through that point. This property does hold and turns out to be characteristic for lineal functions, as stated by the following:

THEOREM 1. An entire function $f\left(z_{1}, \cdots, z_{n}\right)$ is lineal if and only if it vanishes on planes only.

This theorem implies in particular that every entire zero-free function is lineal. As will be shown below, the Weierstrass product representation may be carried over unchanged to functions in $\mathcal{L}^{(n)}$, reducing in the case when $n=1$ to the classical situation. Hadamard's theory of functions of finite order may likely also be so extended.

Noteworthy are the following further concepts. A lineal polynomial

Presented to the Society, December 28, 1951; received by the editors December 11, 1951.

1 This work was performed on a National Bureau of Standards contract with the University of California, Los Angeles and was sponsored (in part) by the Office of the Air Comptroller, USAF, and by the Office of Naval Research. 
$P\left(z_{1}, \cdots, z_{n}\right)$ is called really lineal if it splits into a product of linear functions with only real coefficients. A really lineal polynomial $P\left(z_{1}, \cdots, z_{n}\right)$ is called positively lineal if the coefficients of its linear factors are all greater than or equal to 0 . Thus $z_{1}^{2}+z_{2}^{2}$ is lineal, $z_{1}^{2}-z_{2}^{2}$ is really lineal, while $z_{1} z_{2}$ is positively lineal. Let us denote by $\mathcal{L}_{r}^{(n)}$ the class of entire functions $f\left(z_{1}, \cdots, z_{n}\right)$ which are regular limits of really lineal polynomials and call the elements of $\mathcal{L}_{r}^{(n)}$ really lineal functions. Likewise let $\mathcal{L}_{p}^{(n)}$ denote the class of positively lineal functions which are defined as regular limits of positively lineal polynomials.

While lineal functions of one variable are completely arbitrary entire functions, this is no longer the case for really lineal and for positively lineal functions of one variable. Indeed, a polynomial $P(z)$ of one variable is really lineal if and only if it has only real zeros and only real coefficients and $P(z)$ is positively lineal if and only if it has only real nonpositive zeros and only non-negative coefficients. Regular limits of polynomials of such special nature have been determined by Laguerre and Pólya (see References $[1 ; 2]$ ) with results which may be described as follows:

Theorem of Laguerre and Pólya. 1. $f(z) \in \mathcal{L}_{r}^{(1)}$ if and only if $f(z)$ admits a Weierstrass product representation of the form

$$
\begin{aligned}
f(z)=C e^{-\gamma z^{2}+\delta z} z^{m} & \prod_{\nu=1}^{\infty}\left(1+\delta_{\nu} z\right) e^{-\delta \nu_{\nu}}, \\
& \left(C, \delta, \delta_{\nu} \text { real, } \gamma \geqq 0, \sum \delta_{\nu}^{2}<\infty\right) .
\end{aligned}
$$

2. $f(z) \in \mathcal{L}_{p}^{(1)}$ if and only if $f(z)$ admits a representation of the form

$$
f(z)=C e^{\gamma z} z^{m} \prod_{\nu=1}^{\infty}\left(1+\delta_{\nu} z\right) \quad\left(C, \gamma, \delta_{\nu} \geqq 0, \sum \delta_{\nu}<\infty\right) .
$$

Using the familiar notation $(a, b)=a_{1} b_{1}+\cdots+a_{n} b_{n}$ and $\|a\|^{2}$ $=(a, a)$ for the scalar product and norm in the unitary space of $n$ complex dimensions, we may now describe the classes $\mathcal{L}_{r}^{(n)}$ and $\mathcal{L}_{p}^{(n)}$ by the following theorems.

THEOREM 2. An entire function is really lineal if and only if it admits a representation of the form

$$
f\left(z_{1}, \cdots, z_{n}\right)=\exp \left(-\sum_{1}^{n} \gamma_{\mu \nu} z_{\mu} z_{\nu}+(z, \delta)\right) \prod_{j=1}^{m}\left(z, c_{j}\right)
$$

$$
\cdot \prod_{\nu=1}^{\infty}\left(1+\left(z, \delta_{\nu}\right)\right) e^{-\left(z, \delta_{\nu}\right)}
$$


where all vectors $c_{j}, \delta, \delta_{\nu}$ have real components, the series $\sum_{v=1}^{\infty}\left\|\delta_{\nu}\right\|^{2}$ converges, while the quadratic form $\sum \gamma_{\mu \nu} z_{\mu} z_{\nu}$ has real coefficients and is positive (definite, or semi-definite).

THEOREM 3. An entire function is positively lineal if and only if it admits a representation of the form

$$
f\left(z_{1}, \cdots, z_{n}\right)=e^{(z, \gamma)} \prod_{j=1}^{m}\left(z, c_{j}\right) \prod_{v=1}^{\infty}\left(1+\left(z, \delta_{v}\right)\right),
$$

where all vectors $c_{j}, \gamma, \delta_{y}$ have real, non-negative components, the series $\sum_{v=1}^{\infty}\left\|\delta_{\nu}\right\|$ being convergent.

Notice that if $n=1$, Theorems 2 and 3 reduce, as they should, to the statements of the theorem of Laguerre and Pólya.

2. On functions vanishing on planes only. Let us denote for convenience by $H^{(n)}$ the class of entire functions $f\left(z_{1}, \cdots, z_{n}\right)$ vanishing on planes only. An element $f$ of $H^{(n)}$ may be zero-free, in which case it is of the form

$$
f\left(z_{1}, \cdots, z_{n}\right)=e^{G\left(z_{1}, \cdots, z_{n}\right)} \quad \text { (G entire). }
$$

If it has zero-planes $L \equiv(z, c)=0$, or $L \equiv 1-(z, \delta)=0$, the following facts are readily established: $1 . f$ vanishes on every zero-plane to a certain finite integral order $\mu$, called the multiplicity of the zero-plane, with the property that $f / L^{\mu}$ is entire and nonzero in at least one point of the plane. 2. The totality of the zero-planes of a function in $H^{(n)}$, each counted as often as its multiplicity indicates, form a denumerable set of planes which have no plane of accumulation, i.e., they must recede to infinity. Disregarding for the moment the finitely many zero-planes passing through the origin, we may denote all zero-planes of $f$ by

$$
\left(z, \delta_{v}\right)=1 \quad(\nu=1,2, \cdots) .
$$

Since necessarily $\left\|\delta_{\nu}\right\| \rightarrow 0$, we may so number these planes that their distances $\left\|\delta_{\nu}\right\|^{-1}$ from the origin form a nondecreasing sequence, hence $\left\|\delta_{1}\right\| \geqq\left\|\delta_{2}\right\| \geqq \cdots$.

Now the classical Weierstrass representation goes through without change. Let

$$
P_{1}(u)=1-u,
$$

$$
P_{k}(u)=(1-u) \exp \left(u+\frac{1}{2} u^{2}+\cdots+\frac{1}{k-1} u^{k-1}\right)
$$

$$
(k=2, \cdots) \text {. }
$$


A sequence of positive integers $k$, may always be determined such that the series

$$
\sum_{\nu=1}^{\infty}\left|\left(z, \delta_{\nu}\right)\right|^{k_{\nu}} \leqq \sum_{\nu=1}^{\infty}\left(\|z\| \cdot\left\|\delta_{\nu}\right\|\right)^{k_{\nu}}
$$

converge for all complex vectors $z=\left(z_{1}, \cdots, z_{n}\right)$. (For instance the choice $k_{\nu}=\nu$ will always do.) Denoting by $\left(z, c_{j}\right)=0(j=1, \cdots, m)$ the zero-planes passing through the origin, we see that

$$
\prod_{j=1}^{m}\left(z, c_{j}\right) \cdot \prod_{\nu=1}^{\infty} P_{k_{\nu}}\left(\left(z, \delta_{v}\right)\right)
$$

is an entire function vanishing on the zero-planes of $f$ to the correct order and nowhere else. If we divide this function into $f$ we obtain a zero-free function of the form (5). Thus

$$
f\left(z_{1}, \cdots, z_{n}\right)=e^{G\left(z_{1}, \cdots z_{n}\right)} \prod_{j=1}^{m}\left(z, c_{j}\right) \prod_{\nu=1}^{\infty} P_{k_{\nu}}\left(\left(z, \delta_{\nu}\right)\right)
$$

is a characteristic product representation for the elements of $H^{(n)}$.

3. A proof of Theorem 1. This proof consists of two parts. (i) $f \in \mathcal{L}^{(n)}$ implies that $f \in H^{(n)}$. Let $f \in \mathcal{L}^{(n)}$. If $f$ is zero-free there is nothing to prove. If $f$ vanishes in a point $z_{0}$ we have to show that $f$ vanishes in a plane passing through $z_{0}$. Without loss of generality we may assume that $f(0, \cdots, 0)=0$. Furthermore, without loss of generality we may assume that $f\left(z_{1}, 0, \cdots, 0\right) \not \equiv 0$. Indeed, assuming $f\left(z_{1}, \cdots, z_{n}\right) \not \equiv 0$, let us examine the Taylor expansion of $f$ at the origin:

$$
f=F_{k}+F_{k+1}+\cdots,
$$

where $F_{k}$ is a homogeneous polynomial in the variables $z_{\nu}$, not vanishing identically. If $F_{k}$ has a term in $z_{1}^{k}$, then our assumption $f\left(z_{1}, 0, \cdots, 0\right) \not \equiv 0$ is verified. If $F_{k}$ has no term in $z_{1}^{k}$, we observe that our classes $\mathcal{L}^{(n)}$ and $H^{(n)}$ are clearly invariant with respect to affine transformations of the variables. An appropriate homogeneous transformation

$$
z_{\mu}=\sum_{\nu=1}^{n} c_{\mu \nu} w_{\nu} \quad(\mu=1, \cdots, n)
$$

$\left[F_{k}\left(c_{11}, c_{21}, \cdots, c_{n 1}\right) \neq 0\right.$ is the only requirement] will transform $F_{k}$ into a $k$ th degree form of $w_{1}, \cdots, w_{n}$ having a nonvanishing term in $w_{1}^{k}$. 
It now follows that $f\left(z_{1}, 0, \cdots, 0\right)$ admits $z_{1}=0$ as a $k$-fold zero $(k \geqq 1)$. Let $P_{\nu}\left(z_{1}, \cdots, z_{n}\right)$ be a sequence of lineal polynomials such that reg. $\lim P_{\nu}=f$. But then also reg. $\lim P_{\nu}\left(z_{1}, 0, \cdots, 0\right)$ $=f\left(z_{1}, 0, \cdots, 0\right)$. By Hurwitz's theorem $P_{\nu}\left(z_{1}, 0, \cdots, 0\right)$ has at least $k$ zeros in $\left|z_{1}\right|<\rho$, no matter how small $\rho$ is, provided $\nu>N(\rho)$. Since $P_{\nu}\left(z_{1}, \cdots, z_{n}\right)$ is lineal, surely one of its linear factors, $L_{\nu}$ say, must vanish in a point which is within a $\rho$-neighborhood of the origin. Thus $P_{\nu}\left(z_{1}, \cdots, z_{n}\right)$ vanishes on a plane $L_{\nu}=0$ whose distance to the origin converges to zero. Taking a proper subsequence, if necessary, we may assume that the planes $L_{\nu}=0$ converge to a limit-plane $L=0$. We now claim that $f=0$ on $L=0$. Indeed, let $z=\zeta$ be a point on $L=0$. Then $\zeta=\lim \zeta_{\nu}$, where $\zeta_{\nu}$ is on $L_{\nu}=0$. But $P_{\nu}\left(\zeta_{\nu}\right)=0$ and the regular convergence of $P_{\nu}(z)$ to $f(z)$ implies that $f(\zeta)=\lim P_{\nu}\left(\zeta_{\nu}\right)$ $=0$.

(ii) $f \in H^{(n)}$ implies that $f \in \mathcal{L}^{(n)}$. We know that $H^{(n)}$ is identical with the class of entire functions admitting a representation of the form (8). There remains to show that (8) will always define lineal functions. Now $P_{k}\left(z_{1}\right) \in \mathcal{L}^{(n)}$, as every entire function of a single variable, and therefore also

$$
P_{k_{\nu}}\left(\left(z, \delta_{\nu}\right)\right) \in \mathcal{L}^{(n)} .
$$

Since $\mathcal{L}^{(n)}$ is obviously closed with respect to multiplication and regular convergence, we conclude that the regularly convergent product of (8) is in $\mathcal{L}^{(n)}$. The proof that $f$, as defined by (8), is in $\mathcal{L}^{(n)}$ will be complete as soon as we establish the following

Lemma. If $G\left(z_{1}, \cdots, z_{n}\right)$ is entire, then

$$
f\left(z_{1}, \cdots, z_{n}\right)=e^{G\left(z_{1}, \cdots, z_{n}\right)}
$$

is a lineal function.

Proof. It suffices to prove the lemma for the special case when $G$ is a polynomial: Indeed, if $Q_{n}$ is the section of the Taylor expansion of $G$, then $Q_{n}$ converges regularly to $G$, hence $e^{Q_{n}}$ converges regularly to $f=e^{G}$. But then the lineality of $e^{Q_{n}}$ implies the lineality of its regular limit $f$. Again, by the multiplicative property of $\mathcal{L}^{(n)}$ it suffices to assume that $G$ reduces to a single monomial, hence to prove that

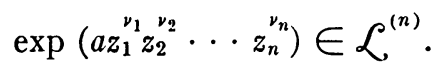

This will readily follow from the fact that a monomial of degree $\nu_{1}+\cdots+\nu_{n}=m$ can always be written as a linear combination with constant coefficients of $m$ th powers of linear forms 


$$
z_{1}^{\nu_{1}} z_{2}^{\nu_{2}} \cdots z_{n}^{\nu_{n}}=c_{1} L_{1}^{m}+\cdots+c_{N} L_{N}^{m} \quad\left(m=\sum \nu_{i}\right) .
$$

In fact the constants $c_{i}$ as well as the coefficients of the variables $z_{\text {, }}$ in the forms $L_{i}$ may be assumed to have rational values.

Taking for granted an identity of the form (10), we indeed conclude that

$$
\exp \left(a z_{1}^{p_{1}} \cdots z_{n}^{\nu_{n}}\right)=\prod_{i=1}^{N} \exp \left(a c_{i} L_{i}^{m}\right)
$$

is lineal, because each factor on the right-hand side is lineal, being an entire function of a linear function of our variables.

To establish an identity of the form (10), we consider first the case of two variables and show that $x^{p} y^{q}$ can be written as

$$
x^{p} y^{q}=\sum_{i=0}^{m} A_{i}\left(x+\lambda_{i} y\right)^{m} \quad(m=p+q),
$$

with rational $\lambda_{i}, A_{i}$. In fact, let $\lambda_{i}=i$; expanding the binomials and comparing coefficients, we obtain for the unknowns $A_{i}$ a linear system of equations whose determinant is the nonvanishing Vandermondian of the $\lambda$ 's. This identity (11) now generalizes automatically for more than two variables. Indeed, let

$$
u^{m} z^{r}=\sum_{j=0}^{m+r} B_{j}\left(u+\mu_{j} z\right)^{m+r}
$$

be the identity corresponding to the monomial $u^{m} z^{r}$. Multiplying (11) by $z^{r}$ we find that

$$
\begin{aligned}
x^{p} y^{q} z^{r} & =\sum_{i=0}^{m} A_{i}\left(x+\lambda_{i} y\right)^{m} z^{r} \\
& =\sum_{i=0}^{p+q} \sum_{j=0}^{p+q+r} A_{i} B_{j}\left(x+\lambda_{i} y+\mu_{j} z\right)^{p+q+r}
\end{aligned}
$$

is also a linear combination of $(p+q+r)$ th powers of linear forms. An obvious induction argument establishes the general validity of (10). This concludes our proof of Theorem 1.

4. A proof of Theorem 2. Let us show first that $f$, as defined by (3), is always in $\mathcal{L}_{r}^{(n)}$, i.e., really lineal. Indeed, since $e^{z_{1}} \in \mathcal{L}_{r}^{(1)}$, we see that $e^{(z, \delta)} \in \mathcal{L}_{\tau}^{(n)}$, provided the vector $\delta$ is real. Thus each of the primary factors of (3) is really lineal and therefore so is their product. There remains to show that 


$$
\exp \left(-\sum_{1}^{n} \gamma_{\mu \nu} z_{\mu} z_{\nu}\right) \in \mathcal{L}_{r}^{(n)}
$$

provided the quadratic form in the exponent is positive. This follows from the representation of the quadratic form as a sum of squares of linear forms:

$$
\begin{aligned}
\exp \left(-\sum \gamma_{\mu \nu} z_{\mu} z_{\nu}\right) & =\exp \left(-L_{1}^{2}-L_{2}^{2}-\cdots-L_{s}^{2}\right) \\
& =\prod_{\sigma=1}^{s} \exp \left(-L_{\sigma}^{2}\right) \quad(s \leqq n),
\end{aligned}
$$

and the remark that $\exp \left(-z_{1}^{2}\right) \in \mathcal{L}_{r}^{(1)}$, hence $\exp \left(-L_{\sigma}^{2}\right) \in \mathcal{L}_{r}^{(n)}$.

Conversely, let $f$ be really lineal and let us show that $f$ must be of the form (3). Now $f \in \mathcal{L}_{r}^{(n)}$ implies that $f \in \mathcal{L}^{(n)}$. By Theorem 1 we conclude that $f$ vanishes on planes only and therefore admits a product representation of the form (8). Let $\beta_{1}, \beta_{2}, \cdots, \beta_{n}$ be real constants, not all zero, and let us consider $f\left(z_{1}, \cdots, z_{n}\right)$ on the ray

$$
z_{1}=\beta_{1} t, z_{2}=\beta_{2} t, \cdots, z_{n}=\beta_{n} t .
$$

Since a really lineal polynomial, considered as a function of $t$ on such a ray, has only real zeros, it is clear that the function

$$
g(t)=f\left(\beta_{1} t, \beta_{2} t, \cdots, \beta_{n} t\right)
$$

belongs to the Laguerre-Pólya class $\mathcal{L}_{\tau}^{(1)}$. If we introduce (12) into the product representation (8) and apply to it the Laguerre-Pólya result that, as a function of $t$, it must be of the form (1), we obtain the following conclusions:

(i) The nonvanishing zeros of $g(t)$, as given by (8) and (12), are furnished by

$$
1-\left(z, \delta_{v}\right) \equiv 1-\left(\beta, \delta_{\nu}\right) t=0 \quad(\nu=1,2, \cdots) .
$$

Since these zeros must all be real, by (1), we conclude that the scalar product $\left(\beta, \delta_{v}\right)$ is real, for each choice of the real $\beta_{1}, \beta_{2}, \cdots, \beta_{n}$. This implies that all vectors $\delta_{\nu}$ are real. Furthermore, (1) also implies that

$$
\sum_{v=1}^{\infty}\left(\beta, \delta_{v}\right)^{2}<\infty
$$

again for every choice of the real vector $\beta$. If $\delta_{\nu}=\left(\delta_{p 1}, \delta_{\nu 2}, \cdots, \delta_{v n}\right)$, on choosing successively $\beta=(1,0, \cdots, 0),(0,1,0, \cdots, 0), \cdots$, $(0, \cdots, 0,1)$, we find that the $n$ series $\sum, \delta_{\nu y}^{2}$ converge, so that 


$$
\sum_{\nu=1}^{\infty}\left\|\delta_{\nu}\right\|^{2}<\infty
$$

But then the series (7) converge if we assume that all $k_{\nu}=2$ $(\nu=1,2, \cdots)$. The canonical product of $(8)$ now becomes of genus unity; writing $-\delta_{\nu}$, instead of $\delta_{\nu},(8)$ reduces to

$$
\begin{aligned}
f\left(z_{1}, \cdots, z_{n}\right)= & e^{G\left(z_{1}, \cdots, z_{n}\right)} \prod_{j=1}^{m}\left(z, c_{j}\right) \\
& \cdot \prod_{\nu=1}^{\infty}\left(1+\left(z, \delta_{\nu}\right)\right) e^{-\left(z, \delta_{\nu}\right)} .
\end{aligned}
$$

(ii) Substituting again (12) into (14) and comparing with (1), we obtain the following identity in $t$

$$
G\left(\beta_{1} t, \cdots, \beta_{n} t\right)=-\gamma t^{2}+\delta t,
$$

for every choice of the $\beta$ 's, where $\gamma$ and $\delta$ are real and depend on the $\beta$ 's only, with $\gamma \geqq 0$. This implies that $G\left(z_{1}, \cdots, z_{n}\right)$ reduces to a real quadratic polynomial

$$
G \equiv-\sum_{\mu, \nu=1}^{n} \gamma_{\mu \nu} z_{\mu} z_{\nu}+\sum_{\nu=1}^{n} \epsilon_{\nu} z_{\nu}
$$

with the property that

$$
\sum \gamma_{\mu \nu} \beta_{\mu} \beta_{\nu}=\gamma \geqq 0,
$$

for arbitrary real $\beta_{1}, \cdots, \beta_{n}$. This entails the positivity of the form.

There remains to show that the homogeneous linear forms $\left(z, c_{j}\right)$ of (3) may all be written with only real coefficients. This we conclude immediately as follows: Observe that we have so far shown that if a really lineal function $f\left(z_{1}, \cdots, z_{n}\right)$ vanishes on a plane which does not contain the origin, such a plane must be real. The same conclusion, however, may be applied also to planes containing the origin, as seen by a shift of the origin to another real point (the property $f \in \mathcal{L}_{r}^{(n)}$ is invariant with respect to real translations).

5. A proof of Theorem 3. If $f \in \mathcal{L}_{p}^{(n)}$, then a fortiori $f \in \mathcal{L}_{r}^{(n)}$ so that we may already assume $f$ to be of the form (3). We now observe that if $P\left(z_{1}, \cdots, z_{n}\right)$ is a positively lineal polynomial and

$$
z_{1}=\beta_{1} t, z_{2}=\beta_{2} t, \cdots, z_{n}=\beta_{n} t\left(\beta_{1} \geqq 0, \cdots, \beta_{n} \geqq 0, \sum \beta_{\nu}>0\right)
$$

is a ray in the positive orthant, then $P\left(\beta_{1} t, \cdots, \beta_{n} t\right)$ is a polynomial in $t$ having only real and nonpositive zeros and non-negative coefficients. Thus $g(t)$, defined by (13), is a function of the Laguerre-Pólya 
class $\mathcal{L}_{p}^{(1)}$. Confronting the product representation for $g(t)$, as given by (3), with what it ought to be in view of (2), we obtain the following conclusions:

(i) The zeros of $g(t)$, given by $1+\left(z, \delta_{v}\right) \equiv 1+\left(\beta, \delta_{v}\right) t=0$, are to be negative. This requires that $\left(\beta, \delta_{v}\right) \geqq 0$ for every choice of the "positive" vector $\beta$. On replacing $\beta$ successively by the unit vectors along the axes we find that all components of $\delta_{\nu}$ are non-negative. Furthermore, (2) also implies that

$$
\sum_{\nu=1}^{\infty}\left(\beta, \delta_{v}\right)<\infty
$$

for every "positive" $\beta$. Thus, if $\delta_{\nu}=\left(\delta_{\nu 1}, \cdots, \delta_{\nu n}\right)$, all $\sum_{\nu=1}^{\infty} \delta_{\nu j}$ converge, so that also

$$
\sum_{\nu=1}^{\infty}\left\|\delta_{\nu}\right\| \leqq \sum_{\nu=1}^{\infty}\left(\delta_{\nu 1}+\cdots+\delta_{v n}\right)<\infty .
$$

The product representation (3) may now be reduced to the form

$$
\begin{aligned}
f\left(z_{1}, \cdots, z_{n}\right)= & \exp \left(-\sum \gamma_{\mu \nu} z_{\mu} z_{\nu}+(z, \delta)\right) \prod_{j=1}^{m}\left(z, c_{j}\right) \\
& \cdot \prod_{\nu=1}^{\infty}\left(1+\left(z, \delta_{\nu}\right)\right) .
\end{aligned}
$$

(ii) Substituting again (15) into (16) and comparing with (2) we obtain

$$
\sum \gamma_{\mu \nu} \beta_{\mu} \beta_{\nu}=0, \quad(\beta, \delta)=\gamma \geqq 0,
$$

for every choice of $\beta_{\nu} \geqq 0$. The quadratic form therefore vanishes and $\delta$ is a "positive" vector.

The final remark to the effect that the vectors $c_{j}$, in (4), may all be chosen so as to have non-negative components is seen as follows. It is clearly sufficient to show that none of the vectors $c_{j}$ can have two components of opposite signs. This, however, is the case, for otherwise we could find positive values $z_{\nu}=p_{\nu}(\nu=1, \cdots, n)$ such that

$$
f\left(p_{1}, \cdots, p_{n}\right)=0 .
$$

On the other hand $f$ is the limit of a sequence of positively lineal polynomials $P_{k}$. (17) implies that

$$
\lim _{t \rightarrow \infty} P_{k}\left(p_{1}, \cdots, p_{n}\right)=0 .
$$


The inequality

$$
\left|P_{k}\left(z_{1}, \cdots, z_{n}\right)\right| \leqq P_{k}\left(p_{1}, \cdots, p_{n}\right) \quad\left(\left|z_{1}\right| \leqq p_{1}, \cdots,\left|z_{n}\right| \leqq p_{n}\right),
$$

shows that (18) implies that $f \equiv 0$.

\section{REFERENCES}

1. E. Laguerre, Sur les fonctions du genre zéro et du genre un, Oeuvres de Laguerre, vol. 1, Paris, 1898, pp. 174-177.

2. G. Pólya, Über Annäherung durch Polynome mit lauter reellen Wurzeln, Rendiconti di Palermo vol. 36 (1913) pp. 1-17.

University of California, Los ANgeles, and

University of Pennsylvania

\section{ON THE EXISTENCE OF GREEN'S FUNCTION}

PETER D. LAX

In this note we shall present a very short proof of the existence of Green's function for Laplace's equation for any domain with sufficiently smooth boundary in any number of independent variables. The proof is based on the continuous dependence of solutions of Laplace's equation on their boundary values. It is a modification of a proof given by Paul Garabedian, see [1]; the difference between the two approaches is that whereas Garabedian operates with a representation of harmonic functions in terms of their boundary data which he obtains by a variational argument, in our argument only the linear and bounded dependence of the solution on the boundary values figures.

1. In this section we shall treat the somewhat simpler two-dimensional case.

We consider a bounded domain $D$ whose boundary $C$ consists of a finite number of smooth curves (i.e., curves with continuous tangents).

$B$ is the Banach space of all continuous functions defined on $C$, normed by the maximum norm.

$B^{\prime}$ is the submanifold of those elements of $B$ for which the boundary value problem can be solved. ${ }^{1}$

Received by the editors December 13, 1951.

${ }^{1}$ It is easy to show that $B^{\prime}$ is closed, but this is not necessary for the argument. 